\title{
THE SULPHONAMIDE TREATMENT OF GONORRHOEA IN WOMEN*
}

\author{
By MAJOR MARJORIE BOLTON, M.B., B.S., R.A.M.C. -
}

For seven years clinicians have been observing and describing the effects of various drugs of the sulphonamide group in the treatment of gonorrhoea. . Most of the treatment has been on out-patients, and various clinics, each with its favourite preparations and dosage, have recorded successful cures. I present a study of the action of these drugs from a different point of view. Instead of gathering a further series of statistics from venereal diseases clinics, I have taken advantage of facilities recently afforded me in the Forces to study the behaviour of this group of drugs on in-patients. In the past eighteen months I have been in charge of seventy-eight beds into which women suffering from venereal diseases are admitted and kept until they are pronounced non-infectious. I have been able to eliminate, therefore, many of the fallacies one usually encounters in attempting to produce statistics. The chief factors through which such errors are made are the following.

(1) The distinction between relapse and reinfection is often impossible to make when the patient is not constantly under observation.

(2) There are not any independent witnesses with out-patients to see that the treatment prescribed has been faithfully carried out. Many female patients vomit the tablets, or find the effects so unpleasant that they do not persist with them, or leave out an occasional dose.

(3) Up to date, all patients treated in an out-patient clinic have been anxious, or at any rate willing, to be cured. The most unmanageable patients either do not attend or soon default.

\section{Method of study in a series of 250 cases}

The 250 cases in the series have been kept under observation day and night for an average period of from three to four weeks and the difficulties just mentioned are therefore eliminated as described below.

(1) During the four weeks the women are in hospital reinfection is not possible and the reappearance of gonococci in the tests during this period is known to be a relapse.

(2) Adequate dosage is ensured by various means. For example, if whole tablets are vomited, they are crushed in milk and given as an emulsion or, in the most tiresome cases, intramuscular injections are given.

(3) The young women in the series were of mixed types between the ages of seventeen and thirty-four. Some were unwilling patients who in civil life would never attend a clinic either for diagnosis or for treatment. They were examined by their medical officers as contacts of infected soldiers, but they themselves denied any symptoms and were antagonistic to treatment. We find that the mentality of this type of woman is such that it is quite impossible to make her understand that intercourse with the infected contact will nullify the effects of any treatment being carried out. When the disease is diagnosed few patients refuse to come into hospital but this type is, nevertheless, a passive obstructor who will still vomit her tablets at the least possible excuse. I feel that of the large number of untreated undiagnosed women with gonorrhoea in Great Britain this type forms the greatest section; and it is also a type very apt to become infected. Such women are, of course, only a minute fraction of the Auxiliary Territorial Service as a whole, and the fact that they come under observation at all is entirely due to the Army system of tracing confacts. Yet in the series presented, although some of the women are not even willing to take their medicine, the rate of cure by sulphonamide drugs is 98 per cent.

The clinic has been run exactly as I would manage an out-patient department. Patients attend the treatment room on specified days and daily treatment is carried

*An address delivered to the Medical Society for the Study of Venereal Diseases, April, 1943. 
out only when prescribed by the medical officer. The sole difference has been that the patients are under control throughout the twenty-four hours and I have been able to give, as routine, a dose which might be a little upsetting for a woman doing her daily work.

Scheme of treatment.-On admission to hospital the patient is examined, routine films and cultures are taken and a wet film examination is made for Trichomonas vaginalis. Occasionally three or four daily consecutive tests are needed before the diagnosis in a clinically suspicious case is confirmed pathologically. It is worth delaying treatment for a few days in some cases if one is suspicious that gonococci should be present in the smears and cultures, as it is then so much easier to impress upon the patient the necessity for treatment. As soon as the diagnosis is made the girl is put to bed and the course of sulphonamide begun. During this time local treatment is carried out only if there is a concurrent trichomonas infection, which in this series occurred in 25 per cent of cases. Tests are taken immediately the sulphonamide course is finished, when the patient is allowed to be up and about. These tests are repeated at approximately four-day intervals, and at the same time the cervix is swabbed clear of mucus with a solution of bicarbonate of soda and the progress of the condition inspected. I find that after treatment in an uncomplicated case there are no longer any clinical signs of infection and the tests are persistently satisfactory. In such cases local treatment is never given.

In my opinion the anatomy of the female pelvic organs is so excellently arranged for drainage that spread of gonorrhoea to the tubes should be unusual. Before the sulphonamide drugs were used I often noticed that an acute salpingitis developed after four or five vigorous endocervical paintings with a silver salt or after some fierce douching. In such cases I was never sure that I had not encouraged the spread of infection. When the sulphonamides became available, however, I decided to give these drugs an opportunity to circulate freely through the cervical blood stream and not to interfere with antiseptic applications. This deliberate policy of non-interference worked so well in practice that I now give local treatment only in cases in which there is present trichomonas vaginitis, a persistent cervical erosion or some unusual complication such as a urethral abscess, salpingitis or some infection of a Bartholin gland.

TABLE I

Results of the Sulphonamide Treatment of Gonorrhoea in Women

\begin{tabular}{|c|c|c|c|}
\hline Treatment & & $\begin{array}{l}\text { No. of } \\
\text { cases }\end{array}$ & $\begin{array}{l}\text { Per cent } \\
\text { cured }\end{array}$ \\
\hline $\begin{array}{lllll}\text { One course of a sulphonamide } & \ldots & \ldots & \ldots & \ldots \\
\text { Two courses of a sulphonamide } & \ldots & \ldots & \ldots & \ldots \\
\text { One course of a sulphonamide and glycerin uterine therapy } \\
\text { Two courses of a sulphonamide and fever treatment } \quad \ldots\end{array}$ & $\begin{array}{l}\cdots \\
\cdots \\
\cdots\end{array}$ & $\begin{array}{r}226 \\
9 \\
10 \\
5\end{array}$ & $\begin{array}{r}90 \cdot 4 \\
3 \cdot 6 \\
4 \cdot 0 \\
2 \cdot 0\end{array}$ \\
\hline TotaL & $\ldots$ & 250 & 100 \\
\hline
\end{tabular}

\section{Results of treatment}

One hundred and nineteen patients required one course of sulphapyridine or sulphathiazole without local treatment.

One hundred and seven had one course of sulphapyridine or sulphathiazole and local treatment for Trichomonas vaginalis or for cervicitis. (Here I would like to draw attention to the fact that the trichomonas retards the ultimate clinical recovery in an acute gonorrhoeal case, and must be treated from the beginning.)

In each of nine cases there was one positive restlt of test after the first course of a sulphonamide, but all the tests were quite satisfactory after the patients had had. a second course of treatment.

Ten patients had salpingitis, mild or severe. This was treated first by a routine course of a sulphonamide then, after dilatation of the cervix, with injection of 
glycerin into the uterus, and finally by a second course of a sulphonamide. There was not any further trouble in these ten cases. This is, of course, treatment which could not be given in an out-patient department, but patients with salpingitis must in any case be treated in bed.

Five patients out of the 250 , that is 2 per cent, gave persistently positive results in tests in both urethra and cervix after heavy doses of the sulphonamides. There were not any clinical complications or obvious pockets of infection. The subsequent immediate cure after fever therapy seemed to indicate that the patients had been infected by a drug-resistant organism from the outset. T.A.B. vaccine was used to produce the fever therapy.

\section{Remote complications}

The above series consists of cases of acute and of the more chronic infections. A few of the patients had transient arthritis in knee or wrist, and two of them had rather severe conjunctivitis, but these conditions cleared up completely with the routine course of a sulphonamide.

I have found the sulphonamide drugs to be of great value in all chronic complications of gonorrhoea. A patient with arthritis of several months' standing will find the pain disappear after an adequate dose of the drug, and the condition is then far more accessible to the physiotherapist. The best results are obtained in the larger joints. The knee is commonly affected in women, possibly due to the kneeling involved in housework, and in such patients the range of movement is greatly improved. I have seen three cases of chronic arthritis of the small joints of the hands in women whose livelihood depended on constant manual dexterity. Pain was always relieved by a course of a sulphonamide and physiotherapy, but the condition soon relapsed. Iritis after gonorrhoea seems to be a rare occurrence in women.

Of all complications salpingitis is the commonest and the one causing most distress if neglected. I feel very strongly that one of the greatest benefits of the sulphonamides in the treatment of gonorrhoea is that an acute salpingitis may be controlled before it reaches a severe or chronic stage. The development of chronic gonococcal salpingitis, such as we used to see, should not occur in the future.

The general rule amongst most gynaecologists was that salpingectomy should be performed when a chronically infected tube fulfilled one of three conditions : (1) chronic pain, (2) chronic muco-purulent discharge, or (3) tests persistently showing gonococci. At the present time sulphonamide therapy and dilatation and glycerin drain will usually improve any of these conditions to the extent that an abdominal operation becomes unnecessary.

\section{Observation after treatment}

Since the introduction of the sulphonamides the "follow-up" period has been gradually shortened. I now trace uncomplicated cases for a period of three to four months only. The experience of the last few years would seem to indicate that a true relapse will be apparent during those months.

A girl leaving a military hospital is in the same position as one who is pronounced cured in a civil V.D. clinic and placed on monthly tests. At present I find that periodic observation and tests for three months are adequate.

Of the returned cases I find that some girls have gone back to the same untreated consort, having failed to ask him to be examined. Some present the usual problem of deciding whether relapse or reinfection has occurred.

The impression gathered is that few patients have a true relapse if they have formed one of the group who react well to a single course of a sulphonamide.

\section{Dosage with sulphonamides}

Until eighteen months ago my routine dosage of sulphanilamide and sulphapyridine for some considerable time had been 1 gramme three times a day for 7 days (totalling 21 grammes). This was for out-patients who took the tablets with few ill effects and only occasional rashes or vomiting. I still use approximately 


\section{THE BRITISH JOURNAL OF VENEREAL DISEASES}

the same dosage but give larger amounts-on the first two days, the total for sulphanilamide and sulphapyridine therapy being 22.5 grammes in six days, that is four tablets, each of 0.5 gramme, three times a day on the first day, three tablets three times a day on the second day, and two tablets three times a day for the next four days: The dosage is always simple so as to avoid errors in administration. Sulphathiazole is given in the following way : 7.5 grammes on the first day and 5 grammes daily for the succeeding four days, totalling 27.5 grammes in five days. These doses are given only in the waking hours and need not in my opinion be spread over the whole twenty-four hours. After the patients have had forty-eight hours' treatment with a sulphonamide, the tests do not show any gonococci but the patients are apt to relapse in three to four days if the drug is discontinued. I feel, therefore, that women do better on a four-day to six-day course than on the very short-period intense courses which they do not tolerate well and which are, in any case, impracticable for out-patients in the ordinary clinics.

\section{Toxic reactions}

Patients are encouraged to take plenty of fluids during the treatment. In spite of the fact that women as a rule have a low fluid intake I have not had any serious case of acetylation. The urine of all patients being treated with sulphonamides is tested qualitatively each day for excretion of the drug.

Case report.-The case report below is one of anuria with unusual features.

During the administration of sulphapyridine ( 22 grammes in seven days) progress had been normal until the seventh day when the patient's temperature rose to $103^{\circ} \mathrm{F}$. in a few hours and she complained of feeling ill. Within twenty-four hours the temperature had fallen below $98^{\circ} \mathrm{F}$. There was deep cyanosis, laboured respiration and an imperceptible rapid pulse, the heart apex.rate was 140 . There was anuria which was due to circulatory failure and not to suppression of renal function. The patient presented a picture of complete failure of the peripheral circulation. She was cold and livid and rapidly becoming moribund. A physician assured me that the failure was in the capillaries and not of cardiac origin. An intravenous drip was set up and three pints of glucose saline run in. There was an amazingly rapid improvement in the patient's general condition. The blood sulphonamide was only 7 milligrams per 100 cubic centimetres when she collapsed. Convalescence lasted many weeks after the damage caused by stasis in the peripheral vessels during the thirty-six hours.

Comparative toxicity.-My experience has been mostly with sulphanilamide, sulphapyridine and sulphathiazole. Undoubtedly sulphapyridine is a great improvement upon sulphanilamide from the clinical point of view, and in the same way sulphathiazole is now tending to replace sulphapyridine. As each new preparation has appeared it has been suggested that it may have a more specific effect on the organism than its predecessor, but my feeling is that of these three the quantity of the drug in the blood stream is a more important factor than its chemical formula. It seems that a minimum concentration of 3 to 4 milligrams pér 100 cubic centimetres of blood is required to ensure that the gonococci will be killed. Therefore the aim in treatment is to produce this concentration with the least distress to the patient. When all my patients were given a routine course of sulphapyridine I found that about 10 per cent developed a profuse rash and considerable malaise on the eighth day from the beginning of treatment, and that a higher percentage vomited in the earlier stages. On the other hand sulphathiazole rarely produces any toxic symptoms; even vomiting is most uncommon although a bigger dose is being given.

Estimations of the sulphonamide concentration in the blood taken in the first forty-eight hours of treatment, with the doses I have mentioned, range between 3 and 8 milligrams per cent. This variation seems to be due to personal idiosyncrasy and cannot be predicted.

\section{Clinical failures}

Clinical failures probably fall into one of two classes: either the patient has living gonococci in a pocket not reached by the blood stream, or the organism is of a resistant strain:

" Pocket" infection.-The first class of patient can be treated easily. A further course of the sulphonamide must be given after the pocket has been drained, 


\section{TREATMENT OF GONORRHOEA}

e.g. a glycerin drain for salpingitis, incision of a Bartholin abscess or massage to empty a urethral abscess.

TABLE II

Gonococcal Sulphonamide-resistance in Infected Partners

(Details of Hospital Treatment and Tests)

\begin{tabular}{|c|c|c|}
\hline & Male Contact & Female Contact \\
\hline Pre-hospital treatment & $\begin{array}{l}\text { Sulphathiazole, } 12 \text { grammes in } \\
2 \text { days }\end{array}$ & Nil \\
\hline On admission to hospital & $\begin{array}{l}\text { Gonococci }+ \\
19 \cdot 5 \text { grammes of sulphapyridine } \\
\text { in } 5 \text { days }\end{array}$ & $\begin{array}{l}\text { Gonococci }+ \\
27 \cdot 5 \text { grammes of sulphapyridine } \\
\text { in } 5 \text { days }\end{array}$ \\
\hline At end of the course & Gonococci + & Gonococci + \\
\hline Further treatment & $\begin{array}{l}\text { Gonococcal vaccine injections } \\
\text { up to } 500 \text { millions : sulpha- } \\
\text { thiazole } 24 \text { grammes in } 4 \text { days }\end{array}$ & $\begin{array}{l}27 \cdot 5 \text { grammes sulphathiazole in } \\
5 \text { days }\end{array}$ \\
\hline At end of the course & Gonococci + & Gonococci + \\
\hline Further treatment & $\begin{array}{c}\text { One fever treatment }\left(103^{\circ} \mathrm{F} .\right) \\
\text { with T.A.B. vaccine }\end{array}$ & $\begin{array}{l}\text { Two fever treatments }\left(106^{\circ} \mathrm{F} \text {. and }\right. \\
104^{\circ} \mathrm{F} \text {.) with T.A.B. vaccine }\end{array}$ \\
\hline After fever therapy & Gonococci + & Gonococci + \\
\hline $\begin{array}{l}\text { Combined fever and sul- } \\
\text { phonamide treatment }\end{array}$ & $\begin{array}{l}\text { Two fever treatments during 24- } \\
\text { gramme course of sulpha- } \\
\text { thiazole : also irrigations }\end{array}$ & $\begin{array}{l}\text { Fever therapy once during 28- } \\
\text { gramme course of sulpha- } \\
\text { thiazole in } 5 \text { days }\end{array}$ \\
\hline After combined treatment & Gonococci negative & Gonococci still + \\
\hline
\end{tabular}

Sulphonamide-resistant organisms.-The second class presents a more difficult problem. There appears to be an increasing number of patients infected by such organisms. Recently one girl who seemed to present an uncomplicated case of gonorrhoea resisted two full courses of sulphathiazole and yet had a blood concentration of 8 milligrams per cent during the first course. At the end of drug treatment her clinical and pathological condition was unchanged. The male contact of this girl presented a resistant case also. He was given four courses of sulphonamide therapy as well as T.A.B. vaccine treatment and has only just been discharged from hospital now that tests have shown him free from infection.

There is a general rule that members of the women's services who are diagnosed as suffering from gonorrhoea shall not be treated by sulphonamides before admission to hospital. This would seem to be an ideal scheme in protecting against the administration of inadequate doses, and in leaving a clear picture for pathological diagnosis. Patients who give vague histories of gonorrhoea and of having taken an indefinite number of tablets are often met with and their explanation is supported by a few clinical signs, the diagnosis being unconfirmed by the bacteriologist. An even greater danger in my opinion is that repeated small doses may in time give the gonococcus some resistance against future treatment by sulphonamides. Moreover, it is becoming apparent that once an organism achieves immunity it retains this property when transmitted to contacts. I have attempted to trace known contacts of the patients who appeared to be drug-resistant, in the hope of finding that they also have been difficult to treat. Three of the five patients whose cases are given in Table I gave a history of the contact being under treatment for a long time, but the girls were unwilling to provide particulars. The fourth case is the one described above (Table II.)

The results of the sulphonamide treatment of gonorrhoea in women is excellent. Sulphathiazole in adequate doses can be taken by out-patients without serious disturbance of their activities. In point of fact many patients tolerate the drug far better when fully occupied than when lying in bed thinking about their symptoms ; also the upright position is an advantage. Up to date the difficult cases have been few, but it will be unfortunate if we produce increasing numbers of drug-resistant strains of the organism.

\section{Venereal Diseases Clinics}

A welcome suggestion by the Minister of Health (Mr. Ernest Brown) has been made, that venereal diseases clinics should employ wherever possible a trained almoner.

Some clinics as we already know employ trained almoners, and their help is invaluable. 


\section{DISCUSSION}

Brig. T. E. Osmond (President) opened the discussion. He inquired about the proportion of gonococcus positive cultures in comparison with similar films taken before and after treatment. Was sulphonamide therapy successful in dealing with closed foci without the aid of local treatment?

Dr. Margaret Rorke referred to the great advantage in treating women as in-patients. With out-patients there was always some uncertainty as to the regularity of dosage when the main treatment was with sulphonamide tablets. Sulphathiazole was tolerated well by her out-patients. Dr. Rorke inquired whether tests of cure were taken at the end of treatment or during or at the end of the monthly period. Were any provocative measures employed ? Sulphathiazole, in her experience, appeared to be effective in the treatment of gonococcal infections which had not responded to or had relapsed after sulphapyridine treatment, but such opinions were unconfirmed without detailed knowledge of how regularly the tablets had been taken by out-patients.

Capt. Eva Gallagher asked how many patients had been treated with sulphapyridine and how many with sulphathiazole. The absence of toxic effects after sulphathiazole treatment was well known, but she had not yet noticed that it gave better results in cases she had dealt with. When taking sulphapyridine patients often vomited and many retired to bed, but this was seldom necessary with sulphathiazole treatment.

Dr. Morna Rawlins expressed satisfaction with a 21-gramme course of treatment in seven days, but had had no experience of a more intense dosage.

Dr. David Nabarro wished that Major Bolton had used only one drug for one patient so that it would have been possible to determine which drug produced the best results. He thought it was now generally recognized that sulphathiazole and sulphadiazine were probably better drugs for the treatment of gonococcus infections than sulphapyridine. Certainly from the patient's point of view these two drugs were more desirable. Those who had taken sulphapyridine knew what an unpleasant drug it was to take; certainly the toxic effects of sulphathiazole did not seem to be so severe, although he believed there was a fatal case of sulphathiazole poisoning reported in the Lancet recently. There was a very good article in the Lancet in April, 1942, which dealt with this particular subject, and mention was made that Miescher wrote that he had reduced the treatment of gonorrhoea to a one-dose treatment, bringing it down from a six-day treatment to a single dose of 10 to 4 half-gramme tablets according to the weight of the patient. It was administered with lime-blossom tea which the Lancet suggested might have increased the absorption of the drug by neutralizing the acid in the stomach. If that were so they were reaching the desired goal at which gonorrhoea could be cured by one dose.

In this connexion he recalled a case at the Hospital for Sick Childrcn, Great Ormond Street where a child attended with a copious discharge in which gonococci were shown to be present by films and by cultures. The child was given one dose of 1 gramme of sulphapyridine and the next day the gonococci had disappeared. The case was observed by Dr. Kathleen Brown for six months and gonococci were never recovered in the vagina, urethra or rectum in smears or culture during the whole of that time. It was astonishing that one dose was effective in curing the disease. After the fifteen months of irrigation which was the practice in his earlier days (although the time was shortened with oestrin treatment) sulphapyridine treatment was surely a remarkable advance.

This was a digression, but he did feel that Major Bolton's results would have been, perhaps, more valuable scientifically if she had used one drug for each particular case ; if one or two courses of sulphapyridine did not clear the patient of infection she might then have tried sulphathiazole or sulphadiazine or T.A.B. vaccine or some other form of heat therapy.

Dr. Kathleen Brown said that recently she had employed for out-patients a 10-gramme course of treatment with sulphathiazole over a period of two days. The toleration of the patients appeared to be variable. The signs of infection appeared to resolve very speedily and local treatment afterwards was often unnecessary. It was too early to ascertain the results of this two-day course, but so far it appeared as satisfactory as a 20-gramme course. Dr. Brown said that she recollected very clearly the little girl referred to by Dr. Nabarro. She had watched the child for six months and considered her to be undoubtedly cured.

Dr. Shanson asked if any member could explain why it was considered important in the treatment of pneumonia to give sulphonamides throughout the night as well as by day and why this spreadover of the dosage appeared unnecessary in the treatment of gonorrhoea.

Lt.-Col. King expressed some difficulty in following Major Bolton's classification of efficiency of sulphathiazole, sulphapyridine and sulphanilamide in that order when the cure rate of the whole series was stated to be 98 per cent. The high figure was surprising for in his hands any type of sulphonamide treatment, even with the addition of fever therapy, had not achieved such a high proportion of good results. If he were fortunate enough to attain such a high cure rate he would look beyond his own technique and wonder whether the right standard of cultural tests had been employed in the assessment of cure of the cases by the pathologist.

He noted that, whereas Major Bolton reported that 250 films were gonococcus positive, there were only 118 instances in which the cultures were positive. This indicated that the diagnostic value of the culture tests used was less than that of the films, whereas the reverse was the usual finding.

With regard to dosage he noted that the courses of sulphonamide treatment were restricted to a five-day period. He agreed that such treatment should not be given over a longer period because more prolonged use produced a high proportion of skin rashes. Patients who developed such rashes were sensitized, sometimes permanently. It seemed unjustifiable to run the risk of develop- 


\section{TREATMENT OF GONORRHOEA}

ment of a state of sensitization when any one of the patients might, in the future, urgently require sulphonamide therapy for some other more acute and serious condition.

With regard to local treatment he agreed that this was probably unnecessary, and in most cases he considered it to be inadvisable to put any medicament into the cervix. He had, however, carried out local daily insufflation of sulphonamide powder which appeared rational. This had not added to the toxic effects.

His patients appeared to suffer from trichomonas infestation more often than those in Major Bolton's series. It appeared more difficult to obtain a cure when these two conditions were present together. The chief difficulty was in eliminating the extremely resistant trichomonas. Lt.-Col. King expressed surprise to hear that Major Bolton thought that the sulphonamides were of value in arthritis and salpingitis. The general impression was that the value of the sulphonamides was very small in these complications which often improved without treatment. The after effects might be severe and he thought that providing the patients were fit fever treatment was then suitable. In the hospital in which he worked groups of 500 patients were treated with courses of sulphonamides over periods varying from three to fourteen days. It was found that there was nothing to choose between treatment over five days and treatment over longer periods such as seven, ten or fourteen days. In the treatment of women sulphapyridine had been used in most cases in courses of 4.5 grammes daily for five days. With this dosage of the drug most of the patients showed severe toxic effects. They were sick, most of them felt ill and had to go to bed, and many could not complete the course of treatment. Since sulphathiazole had been available a course of 6 grammes per day for three days had been used. The toxic reactions had been negligible and the clinical results were excellent.

Capt. Walker, who stated that she had worked with Major Bolton, had followed up many of these patients in the series and had confirmed that the results of the treatment were extremely good. In three cases of salpingitis which she had observed there had not been any clinical signs after treatment.

Major C. S. Nicol asked if any lubricant had been employed before the collection of material for culture purposes by Major Bolton. It was generally held that cultures were more often positive in demonstrating the gonococcus than films, but the presence of a lubricant might interfere with the success of culture. He was not clear as to the ultimate results after T.A.B. vaccine therapy in Major Bolton's cases of resistant gonococcal infection. His own experience, as regards male patients in whom gonococci were shown to be still present in the smears, was that T.A.B. vaccine rarely produced a good result. On the other hand the treatment seemed of value in those patients with persistent signs of inflammation but in whom gonococci could no longer be demonstrated.

Dr. Murray Stuart spoke on the value of sulphadiazine. For the last few months he had treated his patients with sulphadiazine in doses of 4 grammes daily for a period of four days. The results were very satisfactory and there was complete freedom from toxic effects among his patients, including one woman aged 60.

Dr. Clements thought that sulphathiazole was an improvement on the previously available sulphonamides. He obtained excellent results from a two-day treatment of 5 grammes daily and had noted in those patients who progressed well that the response to sulphathiazole had been immediate. He was not in favour of local treatment even when daily baths or sitz baths were available and considered that patients improved just as well when this was omitted.

Dr. R. Forgan, discussing the treatment of arthritis by sulphonamides, thought that it was generally agreed that many cases of gonococcal arthritis did not show any dramatic response to these drugs. Major Bolton, however, had found the results in her cases to be good. Some time ago a paper was published on the results of parenteral administration of a soluble sulphonamide in cases of rheumatoid arthritis. For this condition treatment by sulphonamides by mouth had been disappointing, and the results by injection seemed almost too good to be true. Fuller particulars obtained from the author personally seemed to prove that in such a type of case this soluble sulphonamide, disodium- $p$ - $(\gamma$-phenylpropylamino)-benzene sulphonamide- $\propto, \gamma$-disulphonate, appeared to be particularly effective. It would be interesting to know if any venereologists had tried similar treatment in cases of gonococcal arthritis.

Sqn.-Ldr. Jean Morton said that she had the privilege of carrying out the follow-up in Major Bolton's series of cases, the results in which were excellent. She had used sulphapyridine first as a routine but had been glad to turn to sulphathiazole. At first she advised five-day courses, two daily totals of 6 grammes, then three daily amounts of 5 grammes for three days; in $83 \cdot 1$ per cent of the cases the results were successful. Later, she tried the patients with a three-day course ( 7 grammes, 6 grammes and 5 grammes), the results of which gave 83.9 successes. For failures after the first course of sulphathiazole she advised an intravenous T.A.B. vaccine injection of 15 million organisms and after four hours another of 20 million organisms; another course of sulphathiazole was then given latir. Formerly the T.A.B. vaccine injections were given at forty-eight hour intervals, but the results appeared better when the injections were given at hourly intervals.

She had one case in which the gonococci persistently appeared in the tests and in which there was complete absence of clinical signs ; at present the patient was receiving hyperthermia treatment. Usually with sulphathiazole the patients' tolerations were excellent. They did not have any difficulty in swallowing the tablets and they got out of bed at the end of the three days feeling extremely well. She had not seen any complications; there had been a few instances of skin eruption but there had not been any effects on the blood count or any enlargement of the spleen. She considered that sulphathiazole was an improvement upon sulphapyridine. 
Major J. Marshall said that in regard to the necessity for local treatment for women he had found that the sulphonamides appeared to be just as effective in the absence of local treatment. He had found trichomonas infestation to be of very frequent occurrence, 30-35 per cent of cases, and it was his routine to add Stovarsol treatment for all women with gonorrhoea so that any missed infestations with trichomonas were dealt with.

As to Dr. Nabarro's objection to a change to another member of the sulphonamide compounds, Major Marshall had found that in resistant infections it was always better to effect a change in the sulphonamide compound. Sometimes it had been necessary to make two or three changes in the variety of sulphonamide in order to attain success. With regard to the necessity for local treatment in women out-patients who were being given sulphonamide treatment he had not seen any additional improvement in a series of patients so treated.

Dr. Mary Gordon said that her present treatment for gonorrhoea in women was a course of sulphathiazole of 5 grammes daily over a period of five days. She inquired whether or not Major Bolton advised any special diet during sulphonamide treatment and whether or not these compounds were given after meals.

Wing-Cdr. McElligott said that every woman with relapsed gonorrhoea that he had seen, wherever she had been treated, had always been treated with more than one course of a sulphonamide. This suggested that the case in which more than one adequate course of a sulphonamide compound was required was a case of which to beware. Much had been heard of " cures" following one, two, three or four courses and he began to wonder whether they really happened.

Major Bolton in reply said that she had great confidence in her pathologist and she herself had seen many of the tests made. In cases in which closed foci were present resolution was established in many during sulphonamide treatment but sometimes and especially when pus was present, surgical drainage was needed. As regards the number of cases treated with sulphapyridine and sulphathiazole the change-over was made about half-way through the series; the results were apparently identical. There were not any short-period courses used in the series. All patients received a five-day treatment. She preferred sulphathiazole because it caused less distress to the patient. With regard to the tests for cure patients were tested immediately after a monthly period if they lived within a reasonable distance of the hospital, otherwise they were admitted for a night and a provocative used. Lubricants were not used prior to taking specimens for assessing cure ; the urethra was swabbed with dry swabs and the cervix treated in the same manner.

As regards diet the patients did not receive large quantities of meat when they were not feeling well, but otherwise they were not restricted. Sulphathiazole was given in doses of 7.5 grammes the first day, 5.grammes during the second day, and 1 gramme each day for the remaining three days. Patients who had had sulphonamides previously for gonorrhoea or pneumonia seemed to be tolerant to additional sulphathiazole.

Dr. Shanson had inquired as to why it appeared necessary to give sulphonamides throughout the night as well as by day when treating pneumonia whereas this was not the custom in treating gonorrhoea. Major Bolton presumed that to maintain the high blood level of sulphonamide (10 milligrams per cubic centimetre) necessary for the effective treatment of pneumonia the dosage had to be given round the clock. In gonorrhoea the desired effect appeared to be achieved with a blood level of 3 milligrams per cubic centimetre.

\title{
THE PATHOLOGY OF ARSENO-THERAPY JAUNDICE*
}

\author{
By J. H. DIBLE, M.B., Ch.B., F.R.C.P. \\ Professor of Pathology, British Postgraduate Medical School, London \\ and J. McMICHAEL, M.D., F.R.C.P.Ed. \\ Reader in Medicine, British Postgraduate Medical School, London
}

The evolution of opinion regarding the causation of jaundice occurring during the arsenical treatment of syphilis has passed through several phases. It was quickly recognized that syphilis per se was not the cause, and the occurrence of epidemic waves of the disease indicated that some additional factor was responsible. The dosage or particular arsenical compound seemed to have no relationship to the condition, and in every respect the clinical course of the jaundice closely resembled that of so-called catarrhal jaundice or epidemic hepatitis. By developing the technique of liver biopsy, the Copenhagen workers, Roholm and Iversen (1939), gave us a new method of approach to the problem. We have been able to apply this technique to thirty-five cases of arsenical jaundice and we have in addition studied cases of ordinary epidemic hepatitis and the jaundice which follows serum infusions, which may be regarded as controls. The only biopsy work on arsenical hepatitis other than that of Roholm and Krarup (1940) was

* An address to the Medical Society for the Study of Venereal Diseases, March, 1943. 